\title{
Extracorporeal Membrane Oxygenation in Coronavirus Disease 2019: Experience from a Single Italian Center
}

\author{
Emiliano Gamberini MD², Federico Coccolini PhD², Alessandro Circelli MD, \\ Emanuele Russo MD1 , Marco Benni MD1, Gustavo lacono MD³, \\ Emanuela Giampalma MD ${ }^{4}$ and Vanni Agnoletti PhD ${ }^{1}$
}

\author{
'Anesthesia and Intensive Care Department, "M. Bufalini" Hospital, Cesena, Italy \\ ${ }^{2}$ General, Emergency and Trauma Surgery Department, Pisa University Hospital, Pisa, Italy \\ "Vascular Surgery Department, "M. Bufalini" Hospital, Cesena, Italy \\ ${ }^{4}$ Radiology and Interventional Radiology Department, "M. Bufalini" Hospital, Cesena, Italy
}

\begin{abstract}
The novel coronavirus infection, declared by the World Health Organization (WHO) as coronavirus disease 2019 (COVID-19), has spread rapidly around the world from China. About 15-30\% of COVID-19 affected patients will develop acute respiratory distress syndrome (ARDS) and, in centers where it is routinely used, extracorporeal membrane oxygenation (ECMO) may be necessary to sustain respiratory and circulatory function, as postulated by WHO [1,2]. ARDS can lead to refractory hypoxemic respiratory failure, while the relatively high incidence of shock in most severe COVID-19 patients requiring intensive care unit (ICU) admission is probably associated with bacterial superinfection, multiple organ failure and myocarditis [3,4]. Veno-venous ECMO (VV-ECMO) is the first choice ECMO configuration for isolated severe respiratory failure. Venous blood is withdrawn from the vena cava and pumped through an artificial membrane lung where oxygen $\left(\mathrm{O}_{2}\right)$ is added and carbon dioxide $\left(\mathrm{CO}_{2}\right)$ removed. Blood with more $\mathrm{O}_{2}$ and less $\mathrm{CO}_{2}$ than normal venous blood is then returned to the patient in another site of the vena cava, helping the impaired native lung to perform gas exchanges. Veno-arterial ECMO (VA-ECMO) is a
\end{abstract}

\section{Corresponding author:}

Emiliano Gamberini, Anesthesia and Intensive Care Department, “M. Bufalini" Hospital, Viale Ghirotti 286, 47251 Cesena, Italy. Email: egamberini74@gmail.com

(c) 2020 CC BY 4.0 - in cooperation with Depts. of Cardiothoracic/ Vascular Surgery, General Surgery and Anesthesia, Örebro University Hospital and Örebro University, Sweden different configuration where venous blood is withdrawn and pumped through the artificial membrane lung as in VV-ECMO, but it is returned to the patient through a major artery, thus providing even circulatory support but less effective respiratory support.

ECMO is a highly specialized and expensive form of life support with the potential for significant complications, in particular hemorrhage, thrombosis and embolization, and its role in the management of pandemic COVID-19 infection remains unclear. Together with the lack of skills, a huge outbreak such as that of the COVID-19 exacerbates the limitations of providing ECMO due to the unavailability of devices and skilled healthcare professionals. ECMO cannot be considered for use in the frontline in a pandemic when all resources are stretched, even in adequately equipped centers, and its application should be reserved for patients with a substantial chance of survival [5].

"M. Bufalini" Hospital is a major trauma center and stroke center in the eastern part of the Emilia-Romagna region in Italy, with the Adriatic Sea forming its oriental border. The outbreak in this area has been linear rather than exponential and, so far, the availability of resources has not been a critical issue. The first ICU cases were admitted on March 6th and, up until March 27th, we have treated 30 severely ill COVID-19 positive patients. COVID ICU patients usually require long periods of mechanical ventilation and ICU stay. In order to face this situation, the existing 17-bed ICU was converted to a COVID-ICU and nine additional beds were set up in the adjacent five rooms in the surgical ward and equipped with vacuum, oxygen and compressed air connections, 
ICU monitors, and mechanical ventilators. A new fivebed ICU dedicated to non-COVID patients was created by reopening the old post-operative ICU in order to allow for the unavoidable ICU needs of the residual elective surgical activity, trauma, and neuro-intensive patients. The number of ICU staff was increased by transferring anesthesiologists and anesthesia nurses from the operating theatre where elective surgical activity had been reduced by up to $80 \%$.

These organizational changes put us in a position, both structurally and professionally, to be able to treat two patients with ECMO during this period. VV-ECMO was performed in a patient with severe refractory hypoxemia after four cycles of prolonged prone position ventilation, while extracorporeal cardiopulmonary resuscitation with VA-ECMO was necessary to rescue a patient suffering cardiac arrest as a consequence of septic shock; this was then switched to VV-ECMO $3 \mathrm{~h}$ later because of hemodynamic improvement with persistent severe hypoxemia.

In conclusion, ECMO can be a rescue option in the COVID-19 pandemic only in settings where the outbreak does not overwhelm the available resources. ARDS induced refractory hypoxemia is the most common indication for VV-ECMO, while myocarditis leading to cardiogenic shock, together with bacterial superinfection with septic shock, can be treated with VA-ECMO.

\section{Ethics Statement}

(1) All the authors mentioned in the manuscript have agreed to authorship, read and approved the manuscript, and given consent for submission and subsequent publication of the manuscript.

(2) The authors declare that they have read and abided by the JEVTM statement of ethical standards including rules of informed consent and ethical committee approval as stated in the article.

\section{Conflicts of Interest}

The authors declare that they have no conflicts of interest.

\section{Funding}

The authors received no financial support for the research, authorship, and/or publication of this article.

\section{REFERENCES}

[1] World Health Organization. Clinical management of severe acute respiratory infection when novel coronavirus $(2019-\mathrm{nCoV})$ infection is suspected-interim guidance. https://www.who.int/publications-detail/clinicalmanagement-of-severe-acute-respiratory-infectionwhen-novel-coronavirus-(ncov)-infection-is-suspected. Accessed February 11, 2020.

[2] Barbaro RP, Odetola FO, Kidwell KM, et al. Association of hospital-level volume of extracorporeal membrane oxygenation cases and mortality. Analysis of the extracorporeal life support organization registry. Am J Respir Crit Care Med. 2015;191(8):894-901.

[3] Chen C, Zhou Y, Wang DW. SARS-CoV-2: a potential novel etiology of fulminant myocarditis. Herz. 2020; https://doi.org/10.1007/s00059-020-04909-z

[4] Chen N, Zhou M, Dong X, et al. Epidemiological and clinical characteristics of 99 cases of 2019 novel coronavirus pneumonia in Wuhan, China: a descriptive study. Lancet. 2020;395:507-13.

[5] MacLaren G, Fisher D, Brodie D. Preparing for the most critically ill patients with COVID-19: the potential role of extracorporeal membrane oxygenation. JAMA. 2020; In Press. doi:10.1001/jama.2020.2342 